\title{
Orificios de entrada atípicos por proyectil de arma de fuego en cráneo
}

\author{
Atypical entrances of gunshot wounds in the skull
}

\begin{abstract}
Resumen
Se presentan dos casos cuya rareza, escaso número de publicaciones en la bibliografía, y la posibilidad de diagnóstico erróneo en la práctica forense, hacen que su conocimiento sea relevante al interpretar los hallazgos autópsicos. El orificio de entrada típico por proyectiles de arma de fuego en cráneo presenta biselado a nivel de la tabla interna. Sin embargo, John Coe observó que en determinados casos presentaban biselado externo. En los disparos tangenciales puede producirse una lesión característica denominada "ojo de cerradura", en la cual uno de los extremos de la herida presentará los bordes filosos, típicos del orificio de entrada, mientras que el otro extremo presentará el biselado externo de los orificios de salida.
\end{abstract}

Palabras clave: Biselado externo. Cráneo. Herida arma de fuego. Orificio en ojo de cerradura.

\section{Abstract}

Two cases are discussed, whose rarity, low number of publications in the literature, and the possibility of misdiagnosis in forensic practice, mean that their disclosure is relevant in interpreting the autopsy findings. The typical entrance gunshot wounds in the skull has internal beveling. However, John Coe noted that in some cases had external beveling. A bullet striking the skull at a tangential angle may produce a particular lesion called "keyhole lesion", in which one end will have the sharp edges typical of a wound of entrance, whereas the other end will have external beveling of a wound of exit.

Key words: External beveling. Gunshot wounds. Keyhole lesion. Skull.

\section{Introducción}

El orificio de entrada típico producido por proyectiles de arma de fuego en cráneo presenta biselado a nivel de la tabla interna.

En 1980, Spitz describió que en raras ocasiones el biselado del orificio de entrada se presenta a nivel de la tabla externa del cráneo ${ }^{1}$. Denominó a este fenómeno "keyhole lesion" ("lesión en ojo de cerradura") y los mecanismos involucrados en su producción fueron descritos posteriormente por Dixon en el año $1982^{2}$.

Poco ha cambiado desde que John Coe Ilamara la atención en 1982 acerca de la escasa literatura re- ferente al biselado externo en los orificios de entrada por proyectiles de arma de fuego en cráneo ${ }^{3}$.

El biselado externo puede observarse cuando el proyectil incide el cráneo, tanto perpendicularmente como tangencialmente. Es en este último caso, es decir en los disparos tangenciales, donde se produce el defecto denominado "en ojo de cerradura"3.

Se caracteriza por ser un orificio con un patrón característico, en el que se exhiben a la vez elementos de los orificios de entrada y de salida.

A continuación se presentan dos casos forenses de la Morgue Judicial de Montevideo, en los cuales se observan las características descritas en este tipo de lesión.
D. Mederos Catalano ${ }^{1}$
N. Bazán Hernández

${ }^{1}$ Médico Legista. Profesor Adjunto del Dpto. de Medicina Legal, UDELAR. Médico Forense, Instituto Técnico Forense, Poder Judicial. ${ }^{2}$ Residente de Medicina Legal, Departamento de Medicina Legal, UDELAR.

Correspondencia: Dr. Domingo Mederos Catalano.

E-mail:

dmederos@internet.com.uy

Fecha de recepción: 12.ENE.2011

Fecha de aceptación:

13.FEB.2011 
Figura 1. Esquema representando: a. orificio de entrada en cuero cabelludo;

b. orificio de salida en cuero cabelludo;

c. orificio de entrada en cráneo

d. fragmento de proyectil intracraneano recuperado en región occipital.

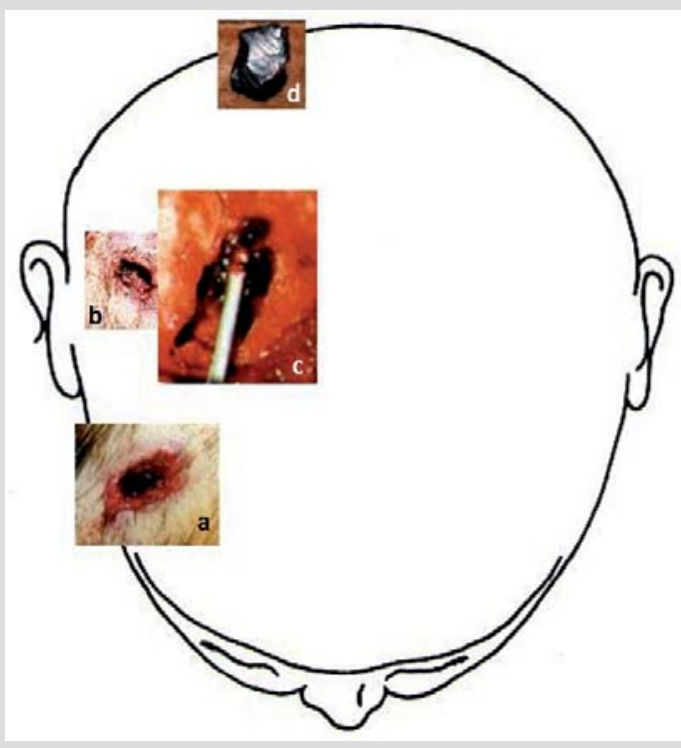

Figura 2 Orificio de entrada en cráneo.

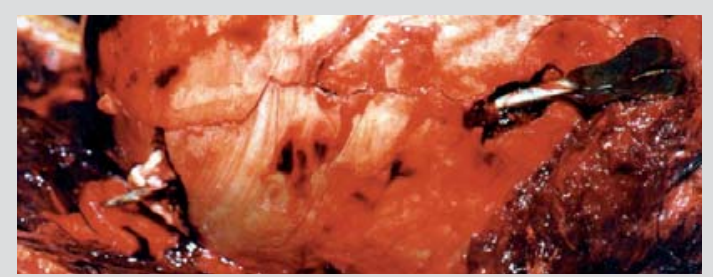

Figura 3. Fragmento de proyecti cortado a bisel.

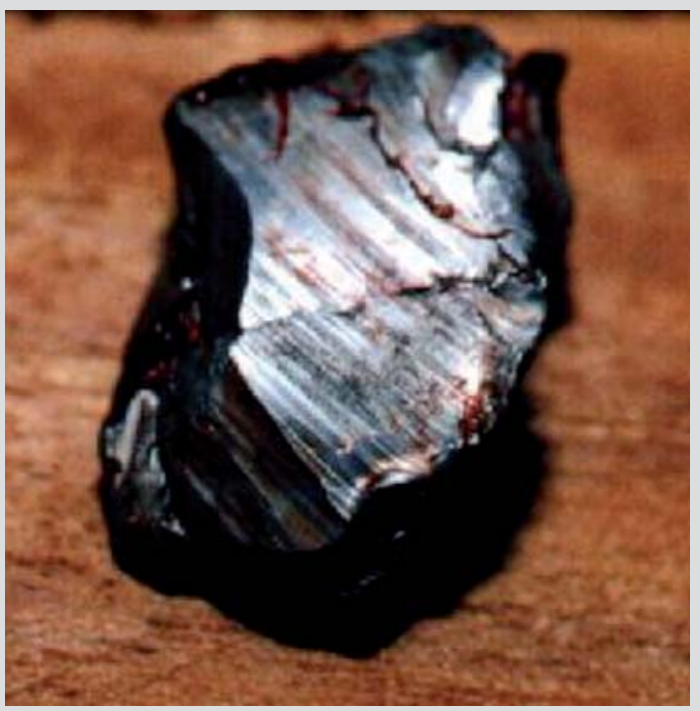

\section{Presentación de casos}

\section{Caso 1}

Hombre de 27 años que durante una persecución policial recibe un disparo de arma de fuego en el cráneo. Trasladado a un centro asistencial fallece a las 24 horas como consecuencia del agravio encefálico. Entre los hallazgos autópsicos se constatan dos orificios en cuero cabelludo (frontal y parietal derechos) y un único orificio de entrada óseo en hueso parietal derecho sin orificio de salida óseo (Figura 1). El orificio de entrada cutáneo, sin tatuaje ni ahumamiento, es catalogado como "lejano" o a "larga distancia". Rebatido el cuero cabelludo, se observa orificio de entrada óseo en región parietal derecha, alargado, comprobándose biselado externo limitado a la zona más posterior del orificio, con trazo de fractura (Figura 2). Se recupera un fragmento del proyectil en región occipital derecha, deformado, de plomo desnudo, calibre .38, con un sector de su superficie "en espejo" (Figura 3). El resto del proyectil produce un orificio de salida cutáneo en región parietal derecha, casi superpuesto al orificio de entrada óseo. El examen histológico confirma la naturaleza de los orificios cutáneos.

\section{Caso 2}

Hombre de 39 años, herida de arma de fuego autoinferida. Ingresó en un centro de asistencia en estado de coma, se realizó TAC que evidenció: swelling bilateral, hematoma subdural agudo en hemisferio izquierdo de $16 \mathrm{~mm}$ de espesor, severo efecto de masa dado por borramiento de surcos, compresión del sistema ventricular, desviación de la línea media $10 \mathrm{~mm}$ y esquirlas metálicas y fragmentos óseos en región parietal izquierda. Es valorado por neurocirujano quién establece que el paciente se encuentra fuera de oportunidad quirúrgica, falleciendo a las 4 horas del ingreso. En la autopsia se comprobó, a nivel de cuero cabelludo, orificio de entrada en región parietal derecha con ahumamiento y tatuaje. Próximo a éste, también en región parietal derecha, orificio de salida (Figura 4). A nivel óseo se constató orificio de entrada, con las típicas características de las lesiones en ojo de cerradura, visualizándose el biselado interno en el sector proximal, y en el distal el biselado externo con trazos de fractura radiados (Figuras 5 y 6 ). Se recuperó fragmento de proyectil de plomo desnudo, cortado a bisel, a nivel de la región parietal izquierda. 


\section{Discusión}

El examen externo de estas heridas puede llevar a confusión, al presentar las características de ambos tipos de lesiones -de entrada y de salida-; por lo cual su conocimiento por parte del examinador se vuelve relevante al momento de interpretar correctamente los hallazgos autópsicos.

El orificio en ojo de cerradura se caracteriza por ser un orificio alargado, donde uno de los extremos de la herida (el proximal) presentará los bordes filosos, típicos de la herida de entrada, mientras que el otro extremo presentará el biselamiento externo típico de los orificios de salida ${ }^{4}$, como observa claramente en los casos presentados (Figura 5).

En este tipo de heridas de arma de fuego con trayectoria tangencial, el proyectil generalmente se fragmenta, exhibiendo un aspecto de corte a bisel. En los dos casos registrados por los autores se recuperaron fragmentos de proyectiles no encamisados con una de sus superficies con dicha característica, es decir con aspecto de "corte a bisel" o "en espejo".

Un fragmento del proyectil se desvía hacia fuera, inmediatamente después de atravesar la tabla externa, originando el biselado externo. Dicho fragmento se desplaza por debajo del cuero cabelludo, pudiendo quedar debajo de él o salir del mismo. Por lo cual, a nivel de cuero cabelludo observaremos dos orificios, uno de entrada y otro de salida, que se encuentran muy próximos entre si, como se puede ver en las Figuras 1 y 4 . El resto del proyectil penetra en la cavidad craneana.

Existen excepciones a esta situación en la cual el proyectil no se fragmenta, sino que penetra intacto en la cavidad craneana; esto ocurre usualmente con proyectiles encamisados. En estos casos, el aspecto de salida del orificio está dado por las ondas de presión provocadas por el impacto del proyectil ${ }^{4}$.

El mecanismo exacto por el cual se producen este tipo de orificios no está completamente explicado, siendo probablemente multifactorial, interviniendo diversos factores como pueden ser: ángulo del disparo; fuerzas de rotación; velocidad, forma y calibre del proyectil; transferencia de energía cinética y resistencia del cráneo ${ }^{5}$.

La forma característica de este tipo de orificios puede explicarse a través de un modelo en el cual existe un vector de fuerza vertical y otro horizontal, los cuales determinan el trauma resultante. El vector de fuerza vertical está dado por el choque del proyectil contra la tabla externa de la bóveda craneana, configurando de esta forma el sector del orificio con las características

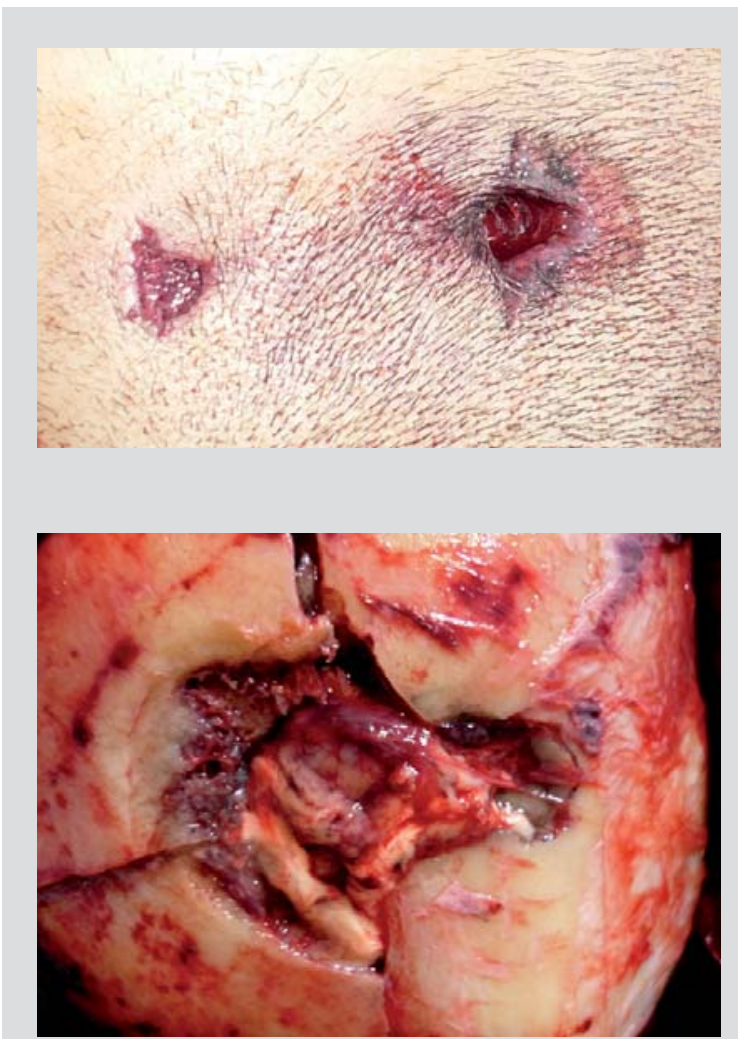

Figura 4.

Orificio de entrada y

salida en cuero cabelludo.

Figura 5.

Lesión típica en

"ojo de cerradura".

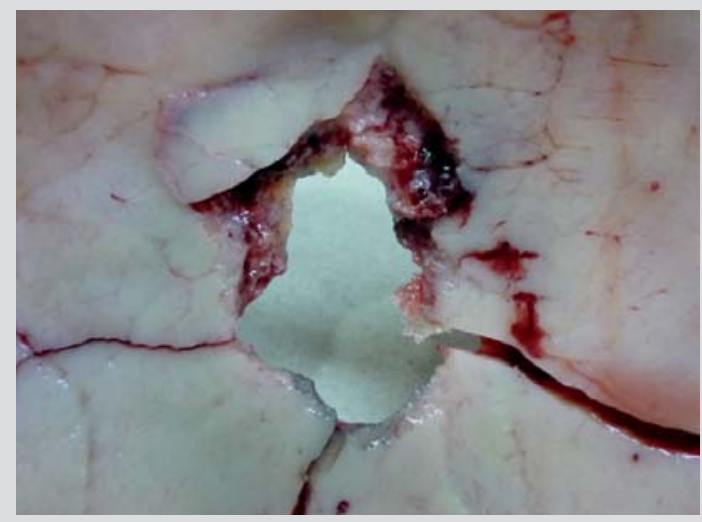

Figura 6.

Lesión en "ojo de cerradura" vista desde la tabla interna del cráneo.

típicas de entrada. Además se producen fracturas secundarias debidas a fragmentos de huesos o del proyectil las cuales se propagan desde el punto inicial del impacto. Esta fuerza vertical proyecta un fragmento de hueso, el cual es empujado hacia arriba. Este segmento de hueso, junto con el fragmento de proyectil que se desvía hacia fuera, representa la fuerza horizontal y es el que produce las características de salida del orificio en ojo de cerradura ${ }^{6}$. 
Esta clase de lesiones puede llevar al médico a creer que en la región intracraneana no se han producido lesiones, ya que puede aparentar una lesión superficial de rozamiento.

\section{Conclusiones}

En ambos casos se trata de un orificio de entrada único (cutáneo y óseo) con fragmentación del pro- yectil, que origina dos trayectos diferentes: uno provoca un orificio de salida cutáneo; y el otro, penetra en el cráneo determinando lesiones encefálicas graves.

La rareza de este tipo de heridas, el escaso número de publicaciones en la bibliografía internacional y la posibilidad de diagnóstico erróneo en la práctica forense, hacen que su conocimiento sea relevante al momento de interpretar los hallazgos autópsicos.

\section{Bibliografía}

1. Spitz WU. Injury by gunfire: gunshot wounds. En: Spitz WU, Fisher RS. Medicolegal investigations of death: guidelines for the application of pathology to crime investigation. Springfield, Illinois: Charles C Thomas 1993;311-412.

2. Dixon DS. Keyhole lesions in gunshot wounds of the skull and direction of fire. J Forensic Sci. 1982;27(3):555-66.

3. Coe JI. External beveling of entrance wounds by handguns. Am J Forensic Med Pathol. 1982;3(3):215-9.
4. Di Maio VJM. An Introduction to the Classification of Gunshot Wounds. En: Di Maio VJM. Gunshot wounds: Practical aspects of firearms, Ballistics and Forensic Techniques. New York: Elsevier 1985;51-98.

5. Baik SO, Uku JM, Sikirica M. A case of external beveling with an entrance gunshot wound to the skull made by a small caliber rifle bullet. Am J Forensic Med Pathol. 1991;12(4):334-6.

6. Jackson AM, Searcey BK, Smirniotopaulos JG, Folio $\mathrm{CL}$. Keyhole Fracture of the Skull. Radiology Corner, November 2008. Disponible en: http://rad. usuhs.mil/amsus/30c_Keyhole_art20_Aug08.pdf. 